\title{
Clinical Significance of Periodic Limb Movement Disorder (PLMD) in Insomnia Patients
}

\author{
Børge Sivertsen*, ${ }^{* 1}$, Siri Omvik ${ }^{1}$, Sivert Straume ${ }^{1}$, Ståle Pallesen ${ }^{2,3}$, Bjørn Bjorvatn ${ }^{3,4}$, Odd E. Havik ${ }^{1}$ \\ and Inger Hilde Nordhus ${ }^{1,3}$
}

${ }^{I}$ Department of Clinical Psychology, University of Bergen, Bergen, Norway, ${ }^{2}$ Department of Psychosocial Science, University of Bergen, Bergen, Norway, ${ }^{3}$ Norwegian Competence Centre for Sleep Disorders, Bergen, Norway, ${ }^{4}$ Department of Public Health and Primary Health Care, University of Bergen, Bergen, Norway

\begin{abstract}
While periodic limb movement disorder (PLMD) in some studies has been linked with a range of negative consequences, others have questioned the validity of the diagnosis. The aim of the present study was to investigate the clinical significance of co-morbid PLMD in terms of daytime impairment in insomnia patients when defined in accordance with the new diagnostic criteria. 64 older adults complaining of insomnia (mean age $=61.9$ ) were included in the study, and assessed with polysomnography, a sleep diary, the Epworth Sleepiness Scale, and a computerized vigilance test. In all, 28 patients had a PLM-index above 15 on at least one polysomnography registration; while the remaining 36 participants served as comparison group (comprising insomniacs patients without co-morbid PLMD). Adjusting for the potential confounding effects of demographics, apnea/hypopneas and symptoms of restless legs syndrome (RLS), we found no significant associations between PLMD and daytime sleepiness (measured with both subjective and objective measures). These findings illustrate that daytime symptoms of insomnia and PLMD may be difficult to distinguish, thus supporting the notion that the clinical significance of PLMD may be questionable.
\end{abstract}

\section{INTRODUCTION}

Alongside its daytime parallel, restless legs syndrome (RLS), periodic limb movement disorder (PLMD) has been linked to a range of negative consequences, including reduced quality of life, work performance, and impaired social and family life [1]. Clinically characterized by repetitive stereotypic leg movements, patients with PLMD typically experience frequent awakenings and loss of deep sleep, which are believed to results in daytime somnolence [2]. The prevalence rates of PLMD increase significantly with age [3]; while epidemiological studies indicate that 4-11\% among the general population suffer from PLMD [4], studies on persons above 65 years have shown that between 11 and $25 \%$ meet the diagnostic criteria [5].

According to the latest edition of the International Classification of Sleep Disorders [6], the diagnosis of periodic limb movements during sleep (PLMS) is defined as episodes of 4 or more limb movements each lasting 0.5 to 5 seconds with amplitudes greater or equal to $25 \%$ of toe dorsiflexion during calibration that occur within 5-90 seconds intervals [6]. If polysomnographic data exceed $15+$ such events per hour on average, and the patient also complain of daytime impairment, a diagnosis of PLMD is warranted.

The fact that many patients are unaware of their limb movements has led to the assumption that PLMS in many cases may be an underlying cause of a complaint of insomnia. Others have argued that PLMS should be regarded as a

*Address correspondence to this author at the Department of Clinical Psychology, University of Bergen, Christiesgt 12, 5015 Bergen, Norway; Tel: +47 55588876; Fax: +47 55589877; E-mail: borge.sivertsen@psykp.uib.no normal phenomenon of old age due to their high frequency [7]. As such, some regard PLMS as clinically insignificant unless they are associated with polyomnographic arousals [8, 9]. In terms of the PLMD-diagnosis, studies examining the association between this condition and other sleep variables [10-15] or daytime functioning yield mixed results $[12,15$, $16]$, thus leaving the clinical significance of PLMD in question $[10-15,17,18]$.

To the best of our knowledge, no studies have examined how PLMD, defined in accordance with the latest diagnostic criteria, is related to daytime impairment [6]. Also, more knowledge is also needed about to what extent PLMD contributes to daytime impairment in insomnia patients. The present study was therefore designed to compare patients with chronic insomnia with and without a co-morbid diagnosis of PLMD, on daytime impairment measured both subjectively and objectively.

\section{METHODS}

\section{Participants and Procedure}

Participants were recruited through newspaper advertisements to a randomized, controlled study of treatment of insomnia [19]; no additional information about the study hypothesis or type of interventions was provided. Inclusion criteria were (1) age 55 years or older, (2) fulfillment of the DSM-IV criteria for insomnia, including difficulties initiating sleep, maintaining sleep, and/or early morning awakenings with no ability of return to sleep, (3) duration of at least 3 months, and (4) complaints of impaired daytime functioning. The following exclusion criteria were used: (1) use of hypnotic medication the last 4 weeks before project start, (2) use of antidepressive or antipsychotic medications, (3) signs 
of dementia or other serious cognitive impairment defined by a score under 23 on the Mini-Mental State Examination [20], (4) presence of a major depressive disorder or other severe mental disorder as identified by a clinical assessment based on The Structured Clinical Interview for DSM-IV (SCID-I) [21], (5) working night shifts and being unable or unwilling to discontinue this work pattern, (6) unwillingness or inability to stop taking sleep medication before start, or (7) having a serious somatic condition preventing further participation.

In all, 64 participants (mean age $=61.9$ years; $s d=6.0$ ) fulfilled the criteria for participating in the present study, of which 29 were women. Included participants were provided with a sleep survey booklet comprising the Epworth Sleepiness Scale [22] and a sleep diary to be completed every morning for 2 weeks [23]. Within this two-week period participants also completed a computerized test of vigilance [24], as well as two nights of polysomnographic monitoring. Two sub-samples were distinguished in accordance with the diagnostic cut-off for PLMD according to the International Classification of Sleep Disorders, second edition [6]. Based on the rationale that PLMS show high night-to night variability [6] and that it is currently recommended to use polysomnography for more than one night in order to make a proper diagnosis [25], we included patients with PLMI $>15$ on at least one of the two registration nights. In all, 28 participants had PLMI above 15 on at least one of the two nights (the PLMD group), while the remaining 36 participants had PLMI equal to or below 15 on both registration nights. Of the total sample, $64.5 \%$ used medication regularly on a daily or weekly basis while the study took place. The most commonly used medication types were antihypertensives $(23.4 \%)$, antithrombotics $(10.9 \%)$, and anticholesterols $(10.9 \%)$. None of the participants had used hypnotics during the last four weeks.

\section{Ethics}

The treatment study from which the current data are collected, was approved by the National Data Inspectorate, the Norwegian Medicines Agency, and The Regional Committee for Medical Research Ethics in Western Norway. Written informed consent was obtained from all participants. Participants received no payment to participate in the study, and they were informed that they could withdraw from the study at any time without stating the reason.

\section{Instruments}

\section{Polysomnography}

Sleep variables were assessed by ambulant clinical polysomnography. All electro-physiologic signals were acquired using Embla A10 (Flaga-Medcare Somnologica ${ }^{\circledR} 3.2$ software package). Data collected during the polysomnography registration included recordings from electroencephalogram, electromyogram, and elctroocculogram. In addition, respiration flow and limb movements of anterior tibialis were recorded, as well as pulse, oximetry and electrocardiogram, body position, and snoring. Sleep stages were scored independently by two trained polysomnography scorers in 30 seconds epochs according to Rechtshaffen and Kales' manual [26]. PLMS was defined as episodes of 4 or more limb movements each lasting 0.5 to 5 seconds that occured with 5-90 seconds intervals and with amplitudes greater or equal to $25 \%$ of toe dorsiflexion during calibration, as outlined in the second edition of the International Classification of Sleep Disorders [6]. The criteria for apnea/hypopnea were defined as: Duration of 10 seconds, a minimum of $50 \%$ in reduction of air flow at the nose and mouth, and a fall in oxygen saturation of at least $3 \%$ or an arousal in the electroencephalogram of more than 3 seconds. PLMI and apnea/hypopnea index (AHI) were calculated for each subject, thus representing the individual's number of periodic limb movement events per hour and number of apneas/hypopneas per hour. In line with common scoring criteria [27], limb movements caused by apnea or hypopnea were not scored as PLMS.

\section{Sleep Disorders Questionnaire}

Symptoms of RLS were assessed by the Sleep Disorders Questionnaire [28]. The questionnaire consists of a number of statements relevant to sleep, and for each statement the participants rate to which degree it applies to them on a 5point scale where 1 indicates "never" and 5 "always". A RLS-index was calculated based on the mean of two items assessing symptoms of RLS (sensations of itchiness or irritability in the legs, and difficulties keeping the legs still).

\section{Sleep Diaries}

Participants completed sleep diaries every morning for two weeks [23], in which one item assessed reported daytime sleepiness on a 5-point scale. The mean rating across the two weeks were used in the current study.

\section{Epworth Sleepiness Scale}

The Epworth Sleepiness Scale [22] is an 8-item selfreport questionnaire designed to assess subjective sleepiness. The patients report on a 4-point scale to what extent they are likely to fall asleep or doze off in different situations. The Cronbach's alpha coefficient was 0.84 in the current sample.

\section{Vigilance (Vigil, 5.0)}

Vigil, test version S1- Quatember-Maly [24], is a 25minute computerized test which registers enduring attention in a situation with few stimuli changes. The subjects are instructed to watch a brightly flashing dot that travels along a circular path in small jumps on the computer screen. When the dot takes a double jump the respondent has to react by pressing a button. Mean reaction time in seconds, and the number of correct responses comprised the two parameters of vigilance performance. The test version used in the present study differentiates between performances that are significantly below average [24].

\section{Statistics}

SPSS 15.0 was used for all statistical analyses. Independent samples t-tests were used to investigate differences on non-categorical outcome variables and participant characteristics, and a chi-square test was used to investigate if the groups differed on the composition of gender. The relationships between PLMD and measures of daytime sleepiness were evaluated by hierarchical regression analyses. To control for possible confounders, age and gender were entered in step 1, while AHI and RLS index were entered in step 2. In the third and final step PLMD (PLMD vs. non-PLMD) was entered. The dependent variables comprised sleepiness 
measured by the sleep diary, the Epworth Sleepiness Scale, and the two vigilance parameters.

\section{RESULTS}

\section{Patient Characteristics}

There was a significantly lower proportion of women in the PLMD group (7 of 28) compared to the non-PLMD group ( 22 of $36 ; \chi^{2}=8.29, d f=64, p<0.01$ ). The PLMD group displayed significantly more wake time after sleep onset and had a higher AHI than the non-PLMD group (see Table 1). No significant group differences were found on age, RLS index, or any of the dependent variables, or on frequency of regular (daily or weekly) use of medications $\left(\chi^{2}\right.$ $=1.69, d f=64, p=0.19)$. There were no differences between the groups on the use of common medications.

\section{Subjective Measures of Sleepiness}

The results from the hierarchical regression analysis showed that sleepiness (measured by the ESS) could not be explained by the variables (Table 2). All together the variables accounted for $8 \%$ of the variance, and adding PLMD in step 3 explained only additionally $0.7 \%$ of the variance. The same pattern was found measuring sleepiness by the sleep diaries; none of the predictors contributed significantly in explaining the variance of this variable (see Table 2).

\section{Objective Measure of Sleepiness}

The predictors entered in step 1, 2 or 3 of the regression analysis did not explain a significant proportion of the variance in the participants' mean reaction time (Table 2). Adding PLMD in step 3 explained only $0.1 \%$ more of the vari- ance compared to the confounders in step 1 and $2(p=0.66)$. In all, $5 \%$ of the total variance was explained.

Age and gender were unrelated to the number of correct responses. However, the AHI and the RLS index were entered in step 2, contributed to a significant increase in the explained variance $(p<0.05)$. The standardized regression coefficient was negative for the AHI implying that an increase on this parameter was associated with a decrease in number of correct responses. For the RLS index, the standardized regression coefficient was positive, thus indicating that a positive relationship between the variables existed. PLMD explained only $0.1 \%$ more of the variance when added in step 3 of the analysis $(p=0.83)$.

\section{PLMI}

Replacing PLMD with PLMI as a predictor variable in step 3 of the hierarchical regression analyses did not cause substantial differences to the results (See Table 3 ).

\section{DISCUSSION}

The aim of the present study was to examine the level of sleepiness and daytime functioning in a group of insomnia patients with and without co-morbid PLMD. In all, we found that the prevalence of PLMD in the current sample was high (44\%), emphasizing the need for investigating how the disorder relates to daytime impairment in insomnia patients [4].

In the current study, PLMD was not significantly associated with increased daytime sleepiness. This finding is strengthened by the fact that common confounding variables, including age, gender, sleep apnea, and RLS, were statistically controlled for. The data can be meaningfully inter-

Table 1. Means and Standard Deviations for the Total Sample, the PLMD ${ }^{\text {a }}$ Group and the Non-PLMD Group and t-Values for the PLMD Group and the Non-PLMD Group at Baseline

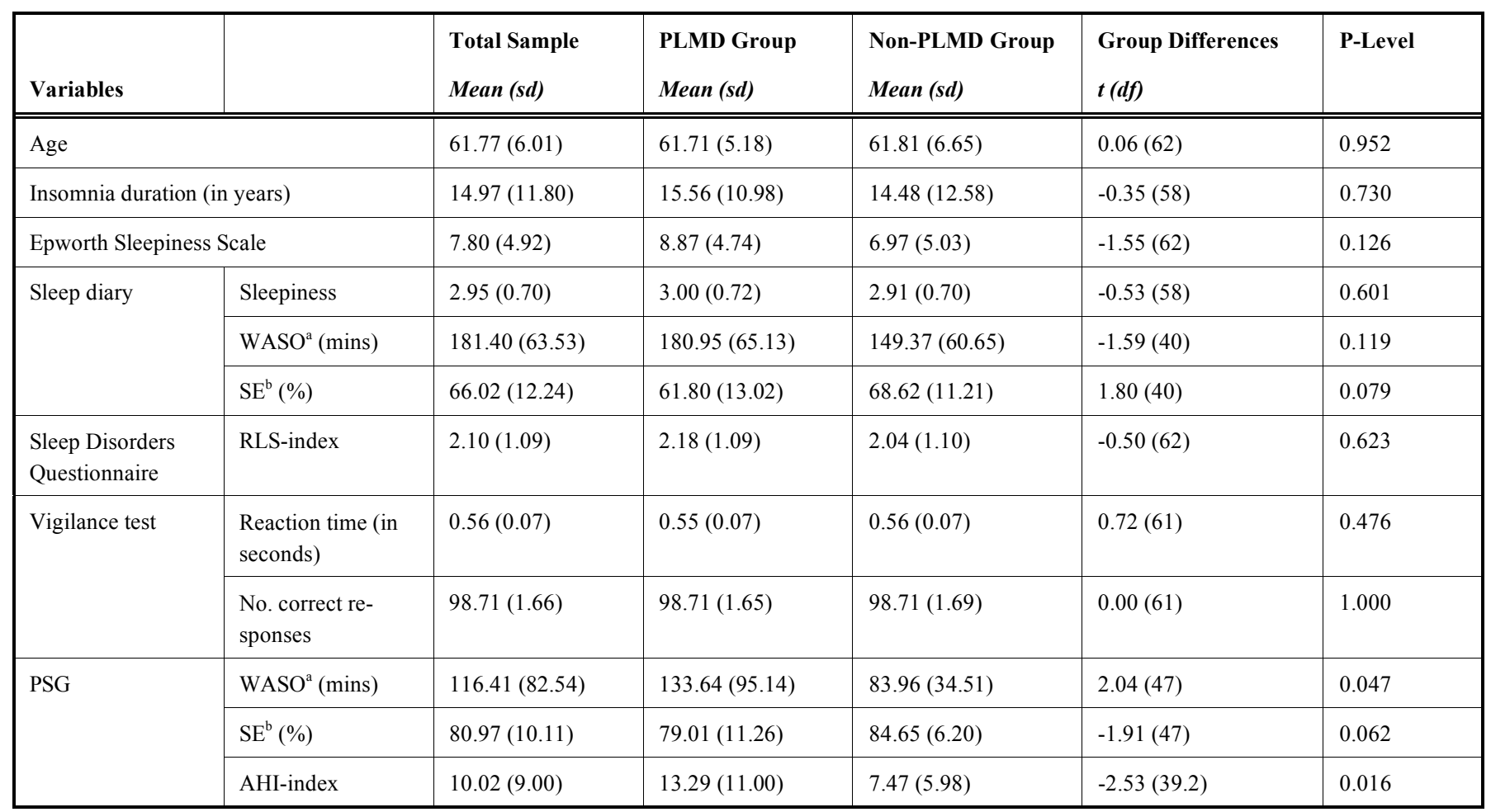

${ }^{a}=$ Wake after sleep onset ${ }^{b}=$ Sleep efficiency. 
Table 2. Hierarchical Regression Analyses Investigating if PLMD Predicts Subjective Sleepiness and Vigilance After Controlling for Third Variables

\begin{tabular}{|c|c|c|c|c|c|c|c|c|c|c|c|c|}
\hline \multirow[b]{2}{*}{ Predictor variables } & \multicolumn{3}{|c|}{$\begin{array}{l}\text { Epworth Sleepiness Scale } \\
\qquad(\mathrm{N}=64)\end{array}$} & \multicolumn{3}{|c|}{$\begin{array}{l}\text { Sleep Diary - Sleepiness } \\
\qquad(\mathrm{N}=60)\end{array}$} & \multicolumn{3}{|c|}{$\begin{array}{l}\text { Mean Reaction Time } \\
\text { (in seconds) }(\mathrm{N}=63)\end{array}$} & \multicolumn{3}{|c|}{$\begin{array}{l}\text { Number of Correct } \\
\text { Responses }(\mathrm{N}=63)\end{array}$} \\
\hline & $\mathrm{R} 2$ & $\Delta \mathrm{R} 2$ & $\beta$ & $\mathrm{R} 2$ & $\Delta \mathrm{R} 2$ & $\beta$ & $\mathrm{R} 2$ & $\Delta \mathrm{R} 2$ & $\beta$ & $\mathrm{R} 2$ & $\Delta \mathrm{R} 2$ & $\beta$ \\
\hline Step 1 & 0.032 & 0.032 & & 0.097 & 0.097 & & 0.046 & 0.046 & & 0.004 & 0.004 & \\
\hline Age & & & -0.092 & & & -0.242 & & & -0.062 & & & 0.157 \\
\hline Step 2 & 0.074 & 0.042 & & 0.128 & 0.030 & & 0.053 & 0.006 & & 0.109 & $0.105^{*}$ & \\
\hline AHI & & & 0.166 & & & 0.190 & & & 0.072 & & & -0.179 \\
\hline RLS index & & & 0.083 & & & 0.048 & & & -0.049 & & & $0.281 *$ \\
\hline Step 3 & 0.081 & 0.007 & & 0.130 & 0.002 & & 0.054 & 0.001 & & 0.110 & 0.001 & \\
\hline
\end{tabular}

* Significant at the 0.05 level (1-tailed).

$\beta=$ standardized regression coefficients.

Table 3. Hierarchical Regression Analyses Investigating if PLMI Predicts Subjective Sleepiness and Vigilance after Controlling for Third Variables

\begin{tabular}{|c|c|c|c|c|c|c|c|c|c|c|c|c|}
\hline \multirow[b]{2}{*}{$\begin{array}{l}\text { Predictor vari- } \\
\text { ables }\end{array}$} & \multicolumn{3}{|c|}{$\begin{array}{l}\text { Epworth Sleepiness Scale } \\
\qquad(\mathrm{N}=64)\end{array}$} & \multicolumn{3}{|c|}{$\begin{array}{c}\text { Sleep Diary - Sleepiness } \\
(\mathbf{N}=60)\end{array}$} & \multicolumn{3}{|c|}{$\begin{array}{l}\text { Mean Reaction Time } \\
\text { (in seconds) }(\mathrm{N}=6 \mathbf{6 3})\end{array}$} & \multicolumn{3}{|c|}{$\begin{array}{l}\text { Number of Correct } \\
\text { Responses }(N=63)\end{array}$} \\
\hline & R2 & $\Delta \mathrm{R} 2$ & $\beta$ & R2 & $\Delta \mathrm{R} 2$ & $\beta$ & $\mathrm{R} 2$ & $\Delta \mathrm{R} 2$ & $\beta$ & $\mathrm{R} 2$ & $\Delta \mathrm{R} 2$ & $\beta$ \\
\hline Step 1 & 0.031 & 0.031 & & 0.109 & $0.107 *$ & & 0.067 & 0.067 & & 0.002 & 0.002 & \\
\hline Age & & & -0.099 & & & 0.215 & & & 0.099 & & & 0.150 \\
\hline Step 2 & 0.073 & 0.042 & & 0.144 & 0.035 & & 0.070 & 0.003 & & 0.114 & $0.112^{*}$ & \\
\hline AHI & & & 0.202 & & & 0.190 & & & 0.059 & & & -0.182 \\
\hline RLS index & & & 0.095 & & & 0.014 & & & -0.014 & & & 0.297 * \\
\hline
\end{tabular}

* Significant at the 0.05 level (1-tailed).

$\beta=$ standardized regression coefficients.

preted in line with the common observation that PLMD often go unnoticed and appear as insomnia [29], demonstrating that the daytime consequences of insomnia and PLMD may be difficult to separate from each other. This points to the necessity of using polysomnographic registrations in the assessment and diagnosing of common sleep disorders, especially since PLMD and insomnia require very different treatment approaches. The findings can also be interpreted as support for those who question the clinical significance of PLMD $[4,12,18]$, as a presence of PLMD made no differences to degree of sleepiness in the current sample.

Although some studies have suggested that PLMS may be associated with sleep-wake complaints, our findings, alongside the majority of studies, point to the contrary. In fact, as early as in 1980 Coleman et al. [30] suggested that there was no evidence that PLMS actually lead to insomnia, and a more recent study on middle-aged and older adults showed little or to relationship between PLMS and impaired sleep (measures both subjectively and with PSG) [31]. Also, a study of younger insomniac patients with and without PLMS concluded that PLMS did not appear to be the primary cause of insomnia in these patients [32]. As such, the present study extend on these findings be also showing that PLMS have little impact on both nocturnal sleep or daytime vigilance.

It should also be mentioned that a possible explanation for PLMS may be the presence of subclinical sleep disordered breathing (SDB), which also was supported in the present study by the higher AHI found in the PLMD group. 
Nevertheless, we find it somewhat surprising that a nighttime problem like PLMD is not associated with impairment in sleepiness, and it remains to be answered why this apparently logical consequence of impaired sleep does not occur. One way to explore the lacking relationship would be to look closer on the implicit assumption that underlies it, namely that a night-time problem like PLMS must cause sleepiness. It is for example possible that PLMS and fragmented sleep in fact do have measurable consequences, but in the shape of autonomous hyperarousal and alertness instead of sleepiness. Or alternatively it may be that a perception of having slept poorly causes individuals to overcompensate in their daytime actions and thereby to perform well on measures of sleepiness. These interpretations are in line with research indicating that exhaustion and fatigue rather than sleepiness is a more likely consequence of sleeplessness [33, 34]. As such, it is possible that the PLMD group might have displayed impairment on other measures of daytime functioning than vigilance and subjective sleepiness.

Finally it has been suggested that higher frequency of periodic limb movements (PLM) with arousal rather than in PLMI may be associated with more disrupted sleep and possibly also more daytime dysfunction [8]. Although one study found no such association with subjective sleepiness [12], the consequences of PLM with arousal for daytime functioning should be investigated further.

\section{Study Limitations}

One limitation of the present study is the limited number of patients, possibly causing lack of adequate power. Despite a difference on the ESS of 1.9 between the non-PLMD and the PLMD, this difference did not reach statistical significance. Based upon the pooled standard deviations on this measure this equals an effect size (Cohen's d) of 0.39. Given the sample sizes and the effect size as well as standard parameters for statistical significance $(\alpha=.05$, two-tailed) this equals a statistical power of 0.33 . Thus, if the difference between two groups in the population is in the magnitude of an effect size of 0.39 then the probability of finding a statistical difference between these two groups given the sample sizes in the present study is $33 \%$. Evidently, this statistical power is low, but this also pertains to the effect size of 0.39 , which is between what Cohen defines as low and a moderate. Also, the reported ESS scores are comparable to what has been reported in previous studies of normal sleepers and PLMD patients $[22,35,36]$, and the difference in means was comparable to what has been observed between severely affected apnoeic patients and sleepers with little or no sleep apnea [37]. Hence, these findings support the view that the Epworth Sleepiness Scale may not be sensitive enough measure of sleepiness in sleep disordered patients [38].

It should also be noted that the participants in the present study performed no poorer than norm samples on number of correct responses on the vigilance test [24]. It is therefore possible that a ceiling effect influenced the results, and that a more demanding vigilance test would have detected differences between the groups. Furthermore it may be objected that that potential confounding effects of medications cannot be ruled out since the participants were not screened for the use of drugs that can contribute to the frequency of PLMS upon entering the study. However, the finding that the groups did not differ in terms of using medications in general strengthens the validity of the results. Also, as the sample consisted of insomnia patients the result may not be generalizable the general population. Nevertheless, PLMS often go unnoticed and subjectively appears as insomnia, and it is therefore likely that the participants represented a typical group of PLMD patients. Finally, the cross-sectional nature of the present study makes it impossible to draw conclusions regarding causality. For example, we cannot determine whether the PLMD is responsible for the insomnia or not, and if treatment would alleviate these symptoms.

\section{CONCLUSIONS}

In the present study, patients fulfilling the latest criteria for PLMD did not differ from insomnia patients without PLMD on neither subjective nor objective indicators of daytime sleepiness. PLMD did in other words not contribute to sleepiness in the insomnia sample, suggesting that daytime symptoms of PLMD and insomnia may be difficult to distinguish. This underlines the necessity of using polysomnographic registrations in the diagnosing of sleep disorders. The findings can also be interpreted in favor of those who challenge the clinical significance of PLMD. More studies are needed to investigate if the results are generalizable to other samples.

\section{ACKNOWLEDGEMENTS}

Funding/support: This research was funded by grants from the University of Bergen, the Meltzer fund, the EXTRA funds from the Norwegian Foundation for Health and Rehabilitation, and the Research Council of Norway. The funding organizations had no role in the design and conduct of the study; collection, management, analysis, and interpretation of the data; and preparation, review, or approval of the manuscript.

\section{ABBREVIATIONS \\ $\mathrm{AHI}=$ Apnea/hypopnea index \\ PLMD = Periodic limb movement disorder \\ PLMI = Periodic limb movements in sleep index \\ PLMS = Periodic limb movements during sleep \\ PLM = Periodic limb movements \\ RLS = Restless legs syndrome}

\section{REFERENCES}

[1] Montplaisir J, Allen R, Walters A, Ferini-Strambi L. Restless legs syndrome and periodic limb movements during sleep. In: Kryger M, Roth T, Dement W, Eds. Principles and practice of sleep medicine, 4th ed. Philadelphia, PA: Elseviers Saunders, 2005; 839-52.

[2] Bonnet M. Acute sleep deprivation. In: Kryger M, Roth T, Dement W, Eds. Principles and Practice of Sleep Medicine. Philadelphia, PA: WB Saunders Company, 2005; 51-66.

[3] Morgan K. Sleep and aging. In: Lichstein KL, Morin CM, Eds. Treatment of late-life insomnia. Thousand Oaks, CA: Sage Publications Inc, 2000; 3-36.

[4] Hornyak M, Feige B, Riemann D, Voderholzer U. Periodic leg movements in sleep and periodic limb movement disorder: prevalence, clinical significance and treatment. Sleep Med Rev 2006; 10: 169-77.

[5] Coleman R, Bliwise NG, Sajben N, et al. Epidemiology of periodic movements during sleep. In: Guilleminault C, Lugaresi E, Eds. Sleep/wake disorders. Natural history, epidemiology and long term evolution. New York, NY: Raven Press, 1988; 217-29. 
[6] American Academy of Sleep Medicine. International classification of sleep disorders: Diagnostic and coding manual. Westchester, IL: Am Acad Sleep Med 2005.

[7] Gehrman P, Stepnowsky C, Cohen-Zion M, et al. Long-term follow-up of periodic limb movements in sleep in older adults. Sleep 2002; 25: 340-3

[8] Leissner L, Sandelin M. Periodic limb movements in sleep: to treat or not to treat? Sleep Med 2002; 3 (Suppl): S27-30.

[9] Rosenthal L, Roehrs T, Sicklesteel J, et al. Periodic movements during sleep, sleep fragmentation, and sleep-wake complaints. Sleep 1984; 7: 326-30.

[10] Youngstedt SD, Kripke DF, Klauber MR, Sepulveda RS, Mason WJ. Periodic leg movements during sleep and sleep disturbances in elders. J Gerontol A Biol Sci Med Sci 1998; 53: M391-4.

[11] Hornyak M, Riemann D, Voderholzer U. Do periodic leg movements influence patients' perception of sleep quality? Sleep Med 2004; 5: 597-600.

[12] Chervin RD. Periodic leg movements and sleepiness in patients evaluated for sleep-disordered breathing. Am J Respir Crit Care Med 2001; 164: 1454-8.

[13] Karadeniz D, Ondze B, Besset A, Billiard M. Are periodic leg movements during sleep (PLMS) responsible for sleep disruption in insomnia patients? Eur J Neurol 2000; 7: 331-6.

[14] Montplaisir J, Michaud M, Denesle R, Gosselin A. Periodic leg movements are not more prevalent in insomnia or hypersomnia but are specifically associated with sleep disorders involving a dopaminergic impairment. Sleep Med 2000; 1: 163-7.

[15] Mendelson WB. Are periodic leg movements associated with clinical sleep disturbance? Sleep 1996; 19: 219-23.

[16] Stepanski E, Lamphere J, Badia P, Zorick F, Roth T. Sleep fragmentation and daytime sleepiness. Sleep 1984; 7: 18-26.

[17] Guilleminault C, Bassiri A. Clinical features and evaluation of obstructive sleep apnea-hypopnea syndrome and upper airway resistance syndrome. In: Kryger M, Roth T, Dement W, Eds. Principles and Practice of Sleep Medicine. Philadelphia, PA: WB Saunders Company, 2005; 1043-52.

[18] Patel S. Restless legs syndrome and periodic limb movements of sleep: fact, fad, and fiction. Curr Opin Pulm Med 2002; 8: 498-501.

[19] Sivertsen B, Omvik S, Pallesen S, et al. Cognitive behavioral therapy vs zopiclone for treatment of chronic primary insomnia in older adults: a randomized controlled trial. JAMA 2006; 295: 2851-8.

[20] Folstein MF, Folstein SE, McHugh PR. "Mini-mental state". A practical method for grading the cognitive state of patients for the clinician. J Psychiatr Res 1975; 12: 189-98.

[21] First MB, Spitzer RL, Gibbon M, Williams JBW. Structural clinical interview for DSM-IV axis I disorders. New York: Biometrics Research Department, 1995.
[22] Johns MW. A new method for measuring daytime sleepiness: the Epworth sleepiness scale. Sleep 1991; 14: 540-5.

[23] Lichstein K, Riedel B. Behavioral assessment and treatment of insomnia: A review with an emphasis on clinical application. Behav Ther 1994; 25: 659-88.

[24] Schuhfried G. Vigilance. Release 25.00. Mödling, Austria: Dr. G. Schuhfried Ges, 2004.

[25] Chesson AL, Jr., Ferber RA, Fry JM, et al. The indications for polysomnography and related procedures. Sleep 1997; 20: 423-87.

[26] Rechtschaffen A, Kales A. A Manual of Standardised Terminology and Scoring System for Sleep Stages of Human Sleep. University of California at Los Angeles: Brain Information Service/Brain Research Institute, 1968.

[27] Butkov N. Polysomnography. In: Lee-Chiong T, Sateia M, Carskadon M, Eds. Sleep medicine. Philadelphia, PA: Hanley and Belfus Inc, 2002; 605-37.

[28] Douglass AB, Bornstein R, Nino-Murcia G, et al. The Sleep Disorders Questionnaire. I: Creation and multivariate structure of SDQ. Sleep 1994; 17: 160-7.

[29] Karatas M. Restless legs syndrome and periodic limb movements during sleep: diagnosis and treatment. Neurologist 2007; 13: 294301.

[30] Coleman R, Pollak C, Weitzman E. Periodic movements in sleep (nocturnal myoclonus): Relation to sleep disorders. Ann Neurol 1980; 8: 416-21.

[31] Karadeniz D, Ondze B, Besset A, et al. Are periodic leg movements during sleep (PLMS) responsible for sleep disruption in insomnia patients? Eur J Neurol 2000; 7: 331-6.

[32] Bastuji H, Garcia-Larrea L. Sleep/wake abnormalities in patients with periodic leg movements during sleep: Factor analysis on data from 24-h ambulatory polygraphy. J Sleep Res 1999; 8: 217-23.

[33] Haba-Rubio J, Staner L, Krieger J, Macher JP. What is the clinical significance of periodic limb movements during sleep? Neurophysiol Clin 2004; 34: 293-300.

[34] Lichstein K, Means M, Noe S, Aguillard R. Fatigue and sleep disorders. Behav Res Ther 1997; 35: 733-40.

[35] Saletu B, Anderer P, Saletu M, et al. EEG mapping, psychometric, and polysomnographic studies in restless legs syndrome (RLS) and periodic limb movement disorder (PLMD) patients as compared with normal controls. Sleep Med 2002; 3 (Suppl): S35-42.

[36] Johns M, Hocking B. Daytime sleepiness and sleep habits of Australian workers. Sleep 1997; 20: 844-9.

[37] Gottlieb DJ, Whitney CW, Bonekat WH, et al. Relation of sleepiness to respiratory disturbance index: the Sleep Heart Health Study. Am J Respir Crit Care Med 1999; 159: 502-7.

[38] Chervin RD. Epworth sleepiness scale? Sleep Med 2003; 4: 175-6.

(c) Sivertsen et al.; Licensee Bentham Open.

This is an open access article licensed under the terms of the Creative Commons Attribution Non-Commercial License (http://creativecommons.org/licenses/by-nc/3.0/) which permits unrestricted, non-commercial use, distribution and reproduction in any medium, provided the work is properly cited. 